\title{
El modus operandi de las organizaciones de productores agropecuarios en Risaralda, Colombia. Primera fase: adaptaciones metodológicas y algunos resultados
}

Carlos Arturo Aristizábal-Rodríguez ${ }^{\star *}$

* Artículo de investigación

** Candidato a MBA con énfasis en Mercadeo. Profesor-investigador de tiempo completo, Universidad Cooperativa de Colombia, sede Pereira, Colombia.

Correo electrónico:

carlos.aristizabalr@campusucc.edu.co

Recibido: 30 de septiembre del 2016 Aprobado: 22 de enero del 2017

Cómo citar este artículo: Aristizábal-

Rodríguez, C. A. (2017). El modus operandi de las organizaciones de productores agropecuarios en Risaralda, Colombia. Primera fase: adaptaciones metodológicas y algunos resultados.

Cooperativismo \& Desarrollo, 111(25), XX-xx. doi: https://doi.org/10.16925/co.v25i111.1878

\section{Resumen}

Propósito: este proyecto de carácter empírico se encuentra actualmente en curso. Su propósito es analizar la forma en que operan 19 asociaciones y cooperativas de productores agrícolas de Risaralda, en cuanto a la estructura y el contenido de cada organización, así como sus estrategias de distribución comercial y fijación de precios. Temas: para el estudio, dichas organizaciones se toman como arreglos interorganizacionales, que a su vez conforman redes entre ellas. Desarrollo: la muestra está conformada por 298 miembros de las organizaciones ya mencionadas, a quienes se les aplica una encuesta; y por los representantes legales de las mismas entidades, a quienes se les realiza una entrevista semiestructurada. Conclusiones: los primeros hallazgos muestran que la mayoría de organizaciones se han conformado con el objetivo de mejorar sus condiciones de negociación frente a terceros, principalmente clientes o intermediarios comerciales, proveedores $y$ agentes del Estado. Frente a este último, ha existido el interés de vincularse al Programa de Apoyo a Alianzas Productivas del Ministerio de Agricultura, el cual se opera a través de las secretarías de agricultura de cada municipio.

Palabras clave: asociaciones y cooperativas agropecuarias, economía social y solidaria, mercado de productos agrícolas, redes de relaciones. 


\title{
Modus operandi of agricultural and livestock producers' organizations in Risaralda, Colombia. First phase: Methodological adaptations and some results
}

\begin{abstract}
Purpose: This empirical project is currently underway. Its purpose is to analyze the way in which 19 agricultural producers' associations and cooperatives of Risaralda operate in terms of the structure and content of each organization, as well as their strategies of commercial distribution and pricing. Themes: For the study, these 19 selected objects of study are taken as interorganizational arrangements, which in turn institute networks between them. Development: The sample is composed by 298 members of the 19 organizations previously mentioned, to whom a survey is applied; and by the legal representatives of the same entities, who are given a semi-structured interview. Conclusions: The first findings show that the majority of these organizations have developed searching to improve their negotiation conditions vis-à-vis third parties, mainly clients or commercial intermediaries, suppliers and state agents. Faced with the latter, interest in being linked to the Ministry of Agriculture's Support Program for Productive Partnerships has emerged. The program is operated through the Department of Agriculture of each municipality.
\end{abstract}

Keywords: agricultural and livestock associations and cooperatives, social and solidarity economy, agricultural products market, relationships networks

\section{O modus operandi das organizações de produtores agropecuários em Risaralda, Colômbia. Primeira fase: adaptações metodológicas e alguns resultados}

\section{Resumo}

Propósito: este projeto de caráter empírico se encontra atualmente em curso. Seu propósito é analisar a forma na qual operam 19 associações e cooperativas de produtores agrícolas em Risaralda (Colômbia) quanto à estrutura e ao conteúdo de cada organização, bem como a suas estratégias de distribuição comercial e fixação de preços. Temas: para o estudo, essas organizações se tomam como ajustes interorganizacionais, que, por sua vez, conformam redes entre elas. Desenvolvimento: a amostra está conformada por 298 membros das organizações já mencionadas, aos quais é aplicado um questionário; também pelos representantes legais das mesmas entidades, com os quais é realizada uma entrevista semiestruturada. Conclusões: os primeiros achados mostram que a maioria de organizações se conformou com o objetivo de melhorar suas condições de negociação com terceiros, principalmente clientes ou intermediários comerciais, fornecedores e agentes do Estado. Ante este último, há o interesse de se vincular ao Programa de Apoio a Parcerias Produtivas do Ministério da Agricultura, o qual é operado por meio das secretarias de agricultura de cada município.

Palavras-chave: associações e cooperativas agropecuárias, economia social e solidária, mercado de produtos agrícolas, redes de relacionamentos. 


\section{Introducción ${ }^{1}$}

En los países en desarrollo la actividad agrícola en pequeña escala está afectada por grandes dificultades. Estos obstáculos traen como consecuencia un aumento en los costos de producción, transporte, distribución y transacción, lo que deriva en condiciones desfavorables para la venta de los productos.

Para ilustrar, los pequeños productores deben enfrentar, además de las condiciones climatológicas (Perry, 2010; Organización de las Naciones Unidas para la Alimentación y la Agricultura-FAO, 2013); distintas plagas y enfermedades fitosanitarias que afectan los cultivos; fluctuaciones de la oferta y la demanda, bajos niveles de producción y productividad (Ramírez y Rozo, 2012); precaria infraestructura física para el manejo de aguas (Perry, 2010; Díaz, Pérez, Lewin, Requena y Oteyza, 2006;); los altos precios de los insumos (Valencia y Mariño, 2014); la difícil topografía del país, asociada al mal estado o ausencia de vías secundarias y terciarias de comunicación (Perry, 2010; Fernández, Hernández e Ibáñez, 2009;); la presencia de un número elevado de intermediarios comerciales (Fernández et al., 2009; Santacoloma, 2011; Otero y Mateus, 2011; Álvarez-Sánchez, Saiz-Vélez, DíazMateus, Castillo-Reyes y Herrera Guzmán, 2012); acceso limitado a fuentes de financiación favorables (Devaux et al., 2009); escasa capacidad empresarial y de liderazgo (Díaz et al., 2006, Álvarez-Sánchez et al., 2012; Cáceres-Gómez, Pardo-Enciso y TorresCárdenas, 2012); ausencia de conocimiento e información sobre el mercado (Devaux et al., 2009); y la falta de confianza entre pares (Perry, 2010; FAO, 2013; Fischer y Quaim, 2012; Poulton, Dorward y Kidd, 2010), entre otros.

La desconfianza entre los productores obstaculiza la realización de procesos de asociatividad (Álvarez-Sánchez et al., 2012; Cáceres-Gómez et al., 2012), y facilita la obtención de altos márgenes de comercialización para los intermediarios, aspecto que obliga a los agricultores a vender sus productos en condiciones desfavorables. Lo anterior se debe, en parte, al escaso poder de negociación de los pequeños productores frente a proveedores, agroindustria, intermediarios y comerciantes mayoristas y detallistas

1. Lo escrito en este artículo se presentó como ponencia en el Encuentro Internacional de Investigación y Buenas Prácticas en Economía Solidaria, realizado el 9 y 10 de noviembre de 2016 en la Universidad Cooperativa de Colombia, sede Bucaramanga.
(Devaux et al., 2009; Kelly, 2012). Como consecuencia, su actividad se ve afectada por bajos niveles de rentabilidad, los cuales ocasionan disminuciones en sus índices de competitividad y redunda en un bajo nivel de vida para los productores.

En el departamento de Risaralda, en algunos casos por iniciativa propia y en su mayoría gracias al Programa de Apoyo a Alianzas Productivas del Ministerio de Agricultura, que inició en el 2002 (Ramírez, 2003), se han creado varias asociaciones y cooperativas de pequeños productores de frutas y hortalizas. Algunas de ellas han sido liquidadas o no operan; en contraste, otras han logrado sostenerse y consolidarse a través de actividades que contemplan el desarrollo de las capacidades, tanto productivas, como administrativas y gerenciales, obteniendo mejores condiciones de negociación ante terceros. Esto se manifiesta en la compra de insumos, la venta de sus productos y otros aspectos tales como la asistencia técnica y el acceso a crédito de bajo costo, lo que trae como consecuencia un aumento en la rentabilidad, la competitividad y en el nivel de vida de los productores.

Según la International Co-operative Alliance (ICA), "una cooperativa es una asociación autónoma de personas unidas voluntariamente para satisfacer sus necesidades y aspiraciones económicas, sociales y culturales en común mediante una empresa de propiedad conjunta y controlada democráticamente" (s. f.).

En Colombia, según la Unidad Administrativa Especial de Organizaciones Solidarias (UAEOS) (2015), las organizaciones de economía social y solidaria se clasifican en organizaciones de economía solidaria y las organizaciones solidarias de desarrollo. Las asociaciones pertenecen al primer grupo, mientras que las cooperativas pertenecen al segundo.

La uAEos (2013) define una asociación como un "ente jurídico sin ánimo de lucro que surge de un acuerdo de voluntades entre dos o más personas vinculadas mediante aportes en dinero, especie o actividad, que puede contraerse a los asociados o a un gremio o grupo social particular" (p.16).

De acuerdo con el Fondo para el Financiamiento del Sector Agropecuario (Finagro) (2013), un pequeño productor es todo aquel quien, incluyendo los del cónyuge, posee activos totales que no superan los 145 salarios mínimos mensuales legales vigentes, los cuales, a pesos del 2014, equivalen a cop 89320000 , y que de este valor, al menos el $75 \%$ esté invertido en el sector agropecuario o que como mínimo las dos terceras partes de sus ingresos sean producto de la actividad agropecuaria. 
Emilio de Velasco (1995) afirma que "el precio es una valoración efectuada sobre un producto y que traducida a unidades monetarias, expresa la aceptación o no del consumidor hacia el conjunto de atributos de dicho producto, atendiendo a su capacidad para satisfacer necesidades" (p. 2). Sin embargo, en el mercado de productos agrícolas es poco lo que puede hacer el pequeño productor a la hora de fijar sus precios, debido a que los precios siempre son impuestos por el mercado.

La distribución comercial comprende todas las actividades necesarias para llevar un producto desde el lugar donde se produce hasta el sitio de consumo (Rangan, Zoltners y Becker, 1986). Estas actividades las desarrolla un grupo de agencias (que pueden ser empresas), o actores que conforman el canal de distribución, el cual está constituido, según Peris, Parra, Lhermie y Romero, (2008) por:

La trayectoria que ha de seguir un bien o servicio desde su punto de origen o producción hasta su consumo, y, además, por el conjunto de personas y/o entidades que permiten la realización de las tareas correspondientes a lo largo de dicha trayectoria. (p. 57)

Varios autores como Buckley (citado por ÁlvarezSánchez et al., 2012) y Simmons y Birchall (2008), proponen la perspectiva de redes para el estudio de las organizaciones de economía social y solidaria. Esto por razones tales como la unión entre la organización y sus miembros, su rica tradición de capital social y humano, su autonomía para servir los intereses de sus miembros y su habilidad para trabajar en red (Simmons y Birchall, 2008). Simmons y Birchall (2008) recomiendan la perspectiva teórica de redes propuesta por Six, Goodwin, Peck y Freeman (2006), quienes proponen un análisis interorganizacional en los principios teóricos fundamentales de las redes de relaciones como son su estructura, contenido, misión, funciones y características institucionales. De esta forma y según lo planteado para esta investigación, se toman las categorías estructura y contenido de la red.

Para comprender mejor los planteamientos de Six et al., (2006), Simmons y Birchall (2008) explican que la estructura comprende subcategorías tales como el grado de centralización de la red constituida, su densidad, la existencia de clústeres y cliques, la equidad estructural, los vínculos de la red y el grado de intermediación existente. El grado de centralización se refiere al nivel de dependencia que tenga la organización alrededor de entes estatales o de agremiaciones. La densidad puede definirse como el grado de desarrollo de la entidad y su voluntad y habilidad para vincularse a otras entidades, a fin de conformar nuevas redes. La existencia de clústeres y cliques al interior o en la periferia de la organización puede hacer la diferencia en la forma que en esta opera. La equidad estructural se refiere al peso que pueda ejercer la organización en su interacción con el Estado. Los vínculos de la red pueden ser fuertes o débiles, dependiendo del nivel de actividad y compromiso de sus miembros. El grado de intermediación depende de la habilidad de algunos integrantes para unir diferentes grupos, transferir conocimientos, ideas y entendimientos dentro la misma organización o con otras.

Por otro lado, Six et al. (2006) mencionan que el contenido se entiende como la naturaleza de los recursos que fluyen a través de la red, que pueden ser tangibles o intangibles. Los recursos tangibles pueden ser dinero o información, mientras que los intangibles pueden ser autoridad o apoyo emocional. Parnell (2001) sugiere que dentro del contenido existen ciertas condiciones que son necesarias para asegurar el desarrollo exitoso de una organización de economía social, los cuales son el acceso a la información sobre la organización, la disponibilidad de fuentes de financiación favorables a sus miembros, contar con mentores que contribuyan a desempeñar actividades de reclutamiento, entrenamiento y desarrollo, y un marco favorable de políticas públicas. Simmons y Birchall (2008) proponen que el flujo de recursos debe cumplir con dos condiciones: la multiplexidad, que corresponde a diversidad de los mismos, y la simetricalidad, entendida como el grado en el cual existe un intercambio equitativo de recursos entre los miembros de la red.

Si se tiene en cuenta la ya expuesta situación de los pequeños productores y los resultados obtenidos por las diferentes asociaciones, se plantean los siguientes interrogantes que permitan analizar dichas organizaciones y tomar elementos que puedan ayudar a mejorar las condiciones organizacionales de las entidades que no han tenido un desempeño satisfactorio:

- ¿Cuál es la forma de operar de las asociaciones y cooperativas de pequeños productores agropecuarios en cuanto a su estructura y contenido?

- ¿De qué manera fijan los precios de sus productos los productores en solitario y las organizaciones en conjunto? 
- ¿Cuáles son las estrategias de distribución comercial que utilizan los campesinos a nivel individual y las organizaciones en colectivo para comercializar sus productos?

\section{Metodología}

La investigación se presenta como de tipo cualitativo. $\mathrm{Su}$ alcance es descriptivo, lo que permite identificar y mostrar el funcionamiento de las organizaciones como redes desde su estructura y contenido, además de la manera en que fijan el precio de sus productos, y los sistemas de distribución comercial que utilizan para poner los mismos en el mercado. Como técnica de recolección de información, se eligió la entrevista con dos variantes, la entrevista estructurada o encuesta y la entrevista semiestructurada.

El estudio comprende dos universos o grupos poblacionales. El primero está conformado por 1169 personas pertenecientes a 19 asociaciones de pequeños productores agrícolas, ubicadas en los municipios de Belén de Umbría, La Celia y Apía, al occidente del departamento de Risaralda. Estas personas son propietarias de predios entre 0,5 y 3 hectáreas, ubicados en la zona rural de dichos municipios; todos son adultos entre los 25 y 70 años de edad, con nivel de escolaridad básico o inferior. El segundo universo, lo integran los 19 representantes legales o presidentes de las mismas entidades, y presentan las mismas características socioeconómicas, con la diferencia de que han liderado los procesos de formación, gestión y consolidación de las organizaciones. Las asociaciones fueron seleccionadas por conveniencia, en razón a la posibilidad reducida de establecer contacto con estas, debido a la dificultad para obtener sus datos. Del primer universo, se extrajo una muestra de 298 personas a través de muestreo aleatorio estratificado, el cual permitió tener en cuenta las diferencias entre las organizaciones estudiadas según el número de sus miembros. Del segundo universo se toma uno de los líderes de cada una de las 19 instituciones, en la mayoría de los casos a los representantes legales.

Dada la dificultad para acceder a los campesinos en sus sitios de residencia, al estar ubicados en zona rural, en veredas y fincas distantes hasta dos horas de las cabeceras municipales, los informantes se seleccionaron por la técnica de bola de nieve, a través de la cual los mismos asociados, o los líderes de las asociaciones refieren a sus compañeros para aplicarles la encuesta en caso de estar presentes en el municipio en el momento de la visita a este, por parte del equipo investigador.
De igual manera, para el segundo grupo poblacional, siguiendo el mismo criterio de conveniencia, se seleccionó a uno de los líderes de cada asociación según su disponibilidad el día de la visita al municipio, dando prioridad a los representantes legales.

Debido a que en la fase anterior del proyecto se comprobó que los miembros de las asociaciones tienen poco conocimiento sobre el funcionamiento de las mismas, se decidió abordarlos a través de encuestas que han sido modificadas en tres oportunidades desde el inicio del estudio, partiendo inicialmente de una prueba piloto. Estas modificaciones han surgido debido a que inicialmente se pretendió abordar la totalidad de categorías del modelo de Six et al. (2006). Sin embargo, al tomar conciencia de la extensión y complejidad de cada categoría, además de la diferencia entre estas, en cuanto a la técnica de recolección de información más apropiada para su estudio, se optó por dividir la investigación en fases, con el fin de abordar al menos dos categorías en cada fase. Adicionalmente, dado que la mayoría de los miembros de las asociaciones tienen nivel de escolaridad de básica primaria o inferior, ha sido necesario adaptar el cuestionario y las diferentes opciones de respuesta para una mayor comprensión por parte de los encuestados.

En contraste, los líderes han sido indagados a través de entrevistas semiestructuradas para aprovechar el mayor conocimiento que tienen de las organizaciones. Para ello, se construyó una guía de entrevista, la cual ha sufrido pocas modificaciones desde el inicio del trabajo de campo, en parte debido a su flexibilidad. Su aplicación ha sido facilitada por la confianza que se ha podido generar, y se ha enriquecido con la experiencia y el conocimiento que tienen la mayoría de los líderes de las instituciones, lo que ha ayudado a hacer más interesantes las entrevistas con inquietudes y temas importantes que han emergido durante su dinámica.

La encuesta pregunta por temas tales como el conocimiento de los costos de producción que tiene el campesino, su cubrimiento a través del precio de venta del producto y la manera en que fijan sus precios. En cuanto a la distribución comercial, se pregunta por los diferentes intermediarios o actores a quienes vende su producto y la relación que tienen con ellos. En cuanto al funcionamiento de cada organización como red, se hacen diferentes preguntas sobre las subcategorías que integran la estructura, además sobre su contenido. Por otra parte, la guía de entrevista contempla las mismas categorías con la diferencia de 
que busca mayor profundidad en los temas, y a la vez con visión global para obtener el panorama completo de las instituciones.

\section{Algunos resultados y discusión}

Al no haberse terminado de realizar la etapa de recolección de los datos, solo puede revelarse información recopilada a través de las entrevistas semiestructuradas realizadas hasta el momento. Esto debido a que es preciso realizar la totalidad de las encuestas para estar en capacidad de utilizar la información que estas arrojen. En este artículo se dará cuenta de los resultados parciales del estudio del contenido de las redes que se explicó anteriormente.

Con respecto al contenido, los entrevistados han manifestado cómo, a través de los vínculos que existen en las organizaciones, fluyen recursos tangibles tales como dinero a través de los fondos rotatorios que tienen constituidos y algunos insumos para sus cultivos como semillas, fertilizantes, plaguicidas y pesticidas. En contraste, las asociaciones que al no cumplir principalmente con el requisito del número mínimo de miembros que exige del Programa de Apoyo a Alianzas Productivas, encuentran muchas dificultades para constituir fondos rotatorios o comunes. Dentro de los recursos intangibles, los entrevistados manifiestan que principalmente fluye el apoyo emocional para los miembros, sobre todo en momentos de incertidumbre, causada más que cualquier cosa por la falta de ingresos de la entidad ocasionada por su inactividad. En consecuencia, al fluir una multiplicidad de recursos, puede decirse que se cumple con la condición de multiplexidad. Por otra parte, los entrevistados afirman que existen condiciones de simetricalidad al haber equidad en el flujo de los recursos; esto se debe, principalmente, a las formas organizacionales adoptadas propias de la economía social y solidaria.

Frente a las condiciones que establece Parnell (2001) para el desarrollo exitoso de las organizaciones de economía social y solidaria, todos los entrevistados manifiestan contar con acceso a fuentes favorables de financiación que provienen, en la mayoría de los casos, de los fondos rotatorios constituidos durante la ejecución de los proyectos pertenecientes al Proyecto de Apoyo a Alianzas Productivas del Ministerio de Agricultura. Con respecto a las políticas públicas, algunos mencionan que se necesita mayor planeación y articulación entre las mismas y menos improvisación por parte del Estado, afirmaciones que son apoyadas por estudios realizados en otros contextos, como el de Galindo-Molina, Gutiérrez-Castro y Trujillo-Nieto (2012); mientras que otros afirman que si existe un marco favorable, y el reto está en poder aprovecharlas de mejor manera. Con respecto a la disponibilidad de mentores que apoyen actividades de reclutamiento, entrenamiento y desarrollo, los entrevistados han sostenido que ellos mismos, acompañados de algunos miembros fundadores, se han encargado de realizar actividades de reclutamiento, mientras que las actividades de entrenamiento y desarrollo han estado soportadas por algunas entidades externas tales como los aliados comerciales, algunas ONG y organismos estatales.

\section{Conclusiones}

Si bien en esta ponencia no se presentan los resultados del análisis de la estructura de las organizaciones analizadas, puede verse cómo se relacionan variables tales como la densidad y el contenido, la equivalencia estructural de los miembros y la condición de simetricalidad, además de la estrecha relación entre la intermediación, y todas las condiciones que debe cumplir el flujo de recursos entre la red. De otra parte, la importancia dada a dichas condiciones se corrobora en la realidad del desarrollo de las entidades materia de estudio.

El estudio de las variables que componen el modelo de Six et al. (2006) amerita un gran esfuerzo metodológico y analítico, dado que muchas de sus subvariables por separado pueden ser materia de un proyecto de investigación individual.

\section{Referencias}

Álvarez-Sánchez, Y., Saiz-Vélez, J., Díaz-Mateus, R., CastilloReyes, D., y Herrera-Guzmán, A. (2012). La Cooperativa de Productores Agropecuarios de Lenguazaque, el Valle de Ubaté y municipios circunvecinos (Coopalac) y su impacto en el desarrollo rural del municipio de Lenguazaque, Cundinamarca. Gestión \& Sociedad, 5(2), 51-73. Recuperado de http://revistas.lasalle.edu.co/ index.php/gs/article/view/2210

Cáceres-Gómez, L., Pardo-Enciso, C., y Torres-Cárdenas, A. (2012). La asociatividad en la dinámica productiva del municipio de El Yopal, Casanare. Gestión \& Sociedad, 5(2), 37-50. Recuperado de http://revistas.lasalle.edu. co/ index.php/gs/article/view/2209 
Devaux, A., Horton, D., Velasco, C., Thiele, G., López, G., Bernet, T. y Ordinola, M. (2009). Collective action for market chain innovation in the Andes. Food Policy, 34(1), 31-38. doi: 10.1016/j.foodpol.2008.10.007.

Díaz, J., Pérez, A., Lewin, P., Requena, B., y Oteyza, S. (2006). Colombia. Nota de análisis sectorial agricultura y desarrollo rural. CAF, FAO. Recuperado de: http://scioteca. caf.com/handle/123456789/655

FAO. (2013). Alianzas público-privadas para el desarrollo de agronegocios

Fernández, M., Hernández, C., Ibáñez, A. M., y Jaramillo, C. (2009). Dinámicas departamentales de pobreza en Colombia 1993-2005. Documento de Trabajo N. ${ }^{\circ} 33$. Programa Dinámicas Territoriales Rurales. Santiago, Chile: Rimisp. Disponible en http://www.rimisp.org/wp-content/files_ mf/1366376788N33_2009_FernandezHernandezIbanezJaramillo_DinamicaspobrezaColombia.pdf

Fondo para el Financiamiento del Sector Agropecuario. (2013). Manual de Servicios de Finagro "Fondo para el Financiamiento del Sector Agropecuario". Versión 25. Recuperado de https://www.finagro.com.co/normas/ manual-de-servicios

Fischer, E., y Qaim, M. (2012). Linking smallholders to markets: determinants and impacts of farmer collective action in Kenya. World Development, 40(6), 1255-1268. doi: https://doi.org/10.1016/j.worlddev.2011.11.018

Galindo-Molina, M., Gutiérrez-Castro, L., y Trujillo-Nieto, L. (2012). Estrategias administrativas y contables de economía solidaria para fortalecer las asociaciones urbanas de agricultores. Gestión y Sociedad, 5(2), 15 36. Recuperado de http://revistas.lasalle.edu.co/index. $\mathrm{php/gs/article/view/2208}$

-Informe de país: Colombia. Estudios de casos de países-América Latina. Roma. Recuperado de http:// www.fao.org/docrep/018/aq437s/aq437s.pdf

International Co-operative Alliance. (s.f.) ¿Qué es una cooperativa? Recuperado de http://ica.coop/en/ what-co-operative

Kelly, S. (2012). Smallholder business models for agribusiness-led development. Roma: Food and Agriculture Organization of the United Nations. Recuperado de http:// www.fao.org/docrep/015/md923e/md923e00.pdf

Otero, M., y Mateus, R. (2011). Un camino al desarrollo territorial: la especialización en la producción de cebolla de rama (Allium fistulosum) en el municipio de Aquitania (Boyacá, Colombia). Gestión \& Sociedad, 4(2), 57-66. Recuperado de http://revistas.lasalle.edu.co/index. php/gs/article/view/242/183
Parnell, E. (2001). The role of cooperatives and other self-help organizations in crisis resolution and socio-economic recovery. Ginebra: ILO. Recuperado de http://staging. ilo.org/public/libdoc/ilo/2001/101B09_544_engl.pdf

Peris, S., Parra, F., Lhermie, C. y Romero, M. (2008). Distribución comercial ( $6^{\mathrm{a}}$ edición). Madrid: Esic. Editorial.

Perry, S. (2010). La Pobreza rural en Colombia. RIMISP, Centro Latinoamericano para el Desarrollo Rural. Disponible en http://www.rimisp.org/wp-content/files_ mf/1366386291DocumentoDiagnosticoColombia.pdf

Poulton, C., Dorward, A. y Kydd, J. (2010). The future of small farms: New directions for services, institutions, and intermediation. World Development, 38(10), 1413-1428. doi: https://doi.org/10.1016/j.worlddev.2009.06.009

Ramírez, M. (2003). Instrumentos e incentivos de política rural: ¿apoyo u obstáculo a una política de tierras? Economía y desarrollo, 2(2). Recuperado de http:// www.fuac.edu.co/download/revista_economica/volumen_2n2/5instrumentos.pdf

Ramírez, L. y Rozo, D. (2012). Diseño de la gestión logística para la cadena productiva de la papa criolla en el municipio de El Rosal Cundinamarca. Caso proveedores de insumos. Gestión \& Sociedad, 5(1), 133-145. Recuperado de http://revistas.lasalle.edu.co/index.php/gs/ article/view/748

Rangan, V. K., Zoltners, A. A. y Becker, R. J. (1986). The channel intermediary selection decision: a model and an application. Management Science, 32(9), 1114-1122. doi: https://doi.org/10.1287/mnsc.32.9.1114

Santacoloma, L. (2011). Análisis comparado de las condiciones de producción de dos asociaciones de productores de fruta del occidente de Colombia para su participación en proyectos de comercio justo. Revista de Investigación Agraria y Ambiental, 2(2), 77-87. doi: https:// doi.org/10.22490/21456453.917

Simmons, R. y Birchall, J. (2008). The role of co-operatives in poverty reduction: Network perspectives. Journal of Socio-economics, 37(6), 2131-2140. Disponible doi: https://doi.org/10.1016/j.socec.2008.04.016

Six, P., Goodwin, N., Peck, E. y Freeman, T., (2006). Managing networks of twenty-first century organizations. Basingstoke: Palgrave Macmillan.

Unidad Administrativa Especial de Organizaciones Solidarias. (2013) ¿Qué es la economía solidaria? Revista Organizaciones Solidarias, Ed. 7. Recuperado de http:// www.orgsolidarias.gov.co/sites/default/files/paginabasica/pdf/Revista\%20Org\%20\%20Solidarias\%207.pdf 
Unidad Administrativa Especial de Organizaciones Solidarias. (2015). ¿Qué es la Unidad Administrativa Especial de Organizaciones Solidarias? Revista Organizaciones Solidarias, 16. Recuperado de http://www. orgsolidarias.gov.co/ sites/default/files/paginabasica/ pdf/01Rev_16_15.pdf
Valencia, M. y Mariño, A. (2014). La empresa agroindustrial colombiana: un análisis de relaciones de poder y configuración de la apropiación de factores productivos. Equidad y Desarrollo, 0(22), 79-97. doi: https://doi. org/10.19052/ ed.3251

De Velasco, E. (1995) El precio: variable estratégica de marketing. Madrid: McGraw-Hill. 